J. RADIAT. RES., SUPPLEMENT, 265-274 (1991)

A Review of Forty-Five Years Study of Hiroshima and Nagasaki Atomic Bomb Survivors

II. BIOLOGICAL EFFECTS

\title{
Persistent Chromosome Aberrations in the Somatic Cells of A-bomb Survivors, Hiroshima and Nagasaki
}

\author{
AKIO A. AWA \\ Cytogenetics Laboratory, Department of Genetics, \\ Radiation Effects Research Foundation, \\ Hiroshima 732, Japan
}

\begin{abstract}
Chromosome aberrations/A-bomb survivors/Dose-response relationship/Lymphocyte chromosomes/Somatic cells
Current status of knowledge on the radiation-induced chromosome aberrations persisting since their induction in 1945 to date in the somatic cells of A-bomb survivors in Hiroshima and Nagasaki is reviewed. Dose-response relationship for chromosome aberration frequencies observed with the use of the old A-bomb dosimetry system (T65D) is also demonstrable based on the new dosimetry system (DS86). Despite the fact that the remarkable decrease in the amount of neutron component relative to the total dose in Hiroshima, there still exist inter-city differences in aberration frequency per unit dose both for kerma and bone marrow dose; the dose-square term is smaller in Hiroshima than in Nagasaki. The differential contribution of neutron radiation may be responsible in some part for the observed difference between Hiroshima and Nagasaki, although proof still remains to be obtained.

There is a wide variability of the frequency of cells with chromosome aberrations between survivors within a given dose range. Random errors in the dose estimates assigned to individual survivors seem responsible, to a large extent, for the observed overdispersions in aberration frequencies in both cities.

New molecular biology-oriented techniques to differentially stain specific chromosomes using fluorescence in situ hybridization with chromosome-specific composite DNA probes seem extremely promising for future rapid, accurate and extensive screening of reciprocal translocations observed predominantly in A-bomb survivors. Such data may be utilized to establish a better biological dosimetry system, especially for those persons who are irradiated in vivo many years before cytogenetic examinations.
\end{abstract}

\section{INTRODUCTION}

In the previous review article ${ }^{1)}$ published in the special issue of this journal in 1975 , emphasis has been placed on (1) the persistence of radiation-induced chromosome damage in the peripheral blood lymphocytes of A-bomb survivors in Hiroshima and Nagasaki, and (2) the difference in the shapes of dose-response curves for chromosome aberration frequencies between the two cities. Some of the major cytogenetic findings in the previous studies will be recapitulated:

(1) Among the radiation-induced chromosome aberrations detectable more than two decades after A-bomb radiation exposure, stable chromosome aberrations, also known as symmetrical exchanges such as reciprocal translocations and inversions, were observed predominantly in survivors of all dose ranges, although it was difficult to detect technically these types of aberrations. 
It is these stable aberrant cells that were the major contributors to the dose-aberration response relationship in A-bomb survivors. In contrast, cells with unstable aberrations, or asymmetrical exchanges such as dicentrics and rings, were less frequent, comprising less than $10 \%$ of the total aberrant cells in the exposed group. This strongly suggested that cells with unstable aberrations would have been selectively eliminated with time after radiation exposure from the circulating lymphocytes by mitosis, assuming that the proportion of stable and unstable aberrations was approximately equal at the time of their induction. Nevertheless, there is still a dose-related increase in unstable aberration frequencies with increasing dose of survivors.

There was a city difference in the frequency of aberrant cells. For each dose group, the aberration frequency was consistently higher in Hiroshima than in Nagasaki. Furthermore, doseresponse curves for each city were different; almost linear in Hiroshima, whereas the dose-square term was much greater in Nagasaki. The observed inter-city difference was then ascribable to the difference in the amount of neutron component relative to the total radiation dose.

\section{RE-ANALYSIS OF CHROMOSOME ABERRATION DATA FOR A-BOMB DOSIMETRY REASSESSMENT}

One of the major objectives since 1975 has been to re-evaluate the chromosome aberration data accumulated to date at RERF on the basis of the new A-bomb dosimetry system termed "DS86").

The present article will review the recent progress in cytogenetic studies of A-bomb survivors, mainly performed at RERF $^{6}$. Emphasis is placed on the difference in the dose-response relationship for thromosome aberration frequencies between booth the new (DS86) and old (T65D) dosimetry systems and Hiroshima and Nagasaki.

\section{MATERIAL AND METHODS}

Blood samples collected between 1968 and 1980 were derived from proximally exposed survivors and their controls, those of either nonexposed (NIC, or not-in-city) or exposed distally, in the RERF Adult Health Study (AHS). Eligible blood samples are those from participants who had not received radiotherapy or radioisotope treatment at any time in the past, and from which a minimum of 30 metaphases per sample were enalyzed, although it was a routine procedure to score 100 metaphases per sample. For some of these individuals with more than one cytogenetic examination, only data from the latest sample were used for the present analysis.

Following the standard procedure blood specimens were cultured for 52 hours, and then cells were processed for chromosome preparations using the conventional Giemsa stain method as described elsewhere ${ }^{3)}$. After 52 hours of incubation time the majority of metaphases observed were found to be in their first in vitro mitosis.

As shown in Table 1, 1,245 subjects from the two cities were examined cytogenetically; 788 (426 exposed and 362 controls) in Hiroshima, and 457 (206 exposed and 251 controls) in Nagasaki. A major reason for the reduction among the Nagasaki survivor sample with relatively high doses 
Table 1. Summary of chromosome aberration data by city and DS86 kerma (Gy)

\begin{tabular}{|c|c|c|c|c|c|c|}
\hline \multirow{2}{*}{$\begin{array}{c}\text { Kerma group } \\
\text { (Gy) }\end{array}$} & \multirow{2}{*}{$\begin{array}{l}\text { Mean } \\
\text { kerma } \\
\text { (Gy) }\end{array}$} & \multirow{2}{*}{$\begin{array}{l}\text { Total } \\
\text { cases } \\
\text { examined }\end{array}$} & \multirow{2}{*}{$\begin{array}{l}\text { Total } \\
\text { cells } \\
\text { counted }\end{array}$} & \multirow{2}{*}{$\begin{array}{l}\text { Total } \\
\text { aberrant } \\
\text { cells }\end{array}$} & \multicolumn{2}{|c|}{ Frequency $(\%)$} \\
\hline & & & & & Mean & S.D. \\
\hline \multicolumn{7}{|c|}{ Hiroshima } \\
\hline \multicolumn{7}{|l|}{ Distally exposed* } \\
\hline $0.000-0.004$ & 0.0 & 362 & 34,299 & 363 & 1.04 & 1.33 \\
\hline \multicolumn{7}{|c|}{ Proximally exposed } \\
\hline $0.005-0.09$ & 0.048 & 13 & 1,188 & 13 & 1.09 & 1.24 \\
\hline $0.10-0.99$ & 0.295 & 60 & 5,624 & 105 & 1.82 & 1.74 \\
\hline $0.50-1.99$ & 0.779 & 90 & 8,697 & 436 & 4.98 & 4.08 \\
\hline $1.00-1.99$ & 1.43 & 152 & 14,500 & 1,309 & 9.00 & 8.09 \\
\hline $2.00-2.99$ & 2.45 & 83 & 8,057 & 1,340 & 16.67 & 11.09 \\
\hline $3.00-3.99$ & 3.37 & 28 & 2,643 & 577 & 21.58 & 12.15 \\
\hline Total & 1.42 & 426 & 40,709 & 3,780 & 9.22 & 9.74 \\
\hline
\end{tabular}

Nagasaki

distally exposed*

0.000-0.004

0.0

251

24,539

345

1.39

1.43

Proximally exposed

$\begin{array}{llrrrrr}0.005-0.09 & 0.70 & 19 & 1,853 & 22 & 1.16 & 1.34 \\ 0.10-0.49 & 0.299 & 48 & 4,584 & 83 & 1.75 & 2.68 \\ 0.50-0.99 & 0.738 & 49 & 4,768 & 140 & 2.87 & 2.91 \\ 1.00-1.99 & 1.42 & 61 & 5,990 & 433 & 7.42 & 7.90 \\ 2.00-2.99 & 2.39 & 23 & 2,215 & 369 & 16.25 & 12.70 \\ 3.00-3.99 & 3.45 & 6 & 600 & 111 & 18.50 & 14.12 \\ \text { Total } & 1.04 & 206 & 20,010 & 1,158 & 5.75 & 8.36\end{array}$

Only samples from individuals with total DS86 kerma 4 Gy are included.

S.D.: standard deviation. * Distally exposed group includes not-in-city subjects.

is that the DS86 system does not currently include procedures for the estimation of doses for individuals exposed in factories or who were shielded by terrain. For each sample, the statistical analysis on cytogenetic data is based on the total number of cells examined (usually 100 metaphases per sample) and the number of cells in which at least one stable chromosome aberration was observed. 


\section{RESULTS}

Table 1 contains information on the distribution of the number of samples, cells scored, and cells with aberrations by kerma for each city. Since the number of cells per individual is not constant, although for the majority of samples 100 metaphases were the basis for this analysis, the mean percentage of cells with chromosome aberrations is not equal to the ratio of the number of cells with aberrations to the total number of cells scored in the dose category (simple mean rate).

The plots in Figure 1 summarize the frequency of cells with chromosome aberrations as a function of kerma estimates for both the old and new dosimetries. The points shown are the unweighted average percentage of cells with aberrations in each dose group plotted as the cityspecific average doses. For each city, the zero dose group includes the samples from all survivors with dose estimates less than $5 \mathrm{mGy}$ and from individuals in the NIC group. Data from samples for all survivors with kerma estimates in excess of $4 \mathrm{~Gy}$ were included in these graphs.

In each of these plots, the aberration frequency appears to be a concave upward function of dose (kerma) with the rate for a given dose (kerma) being generally lower in Nagasaki than
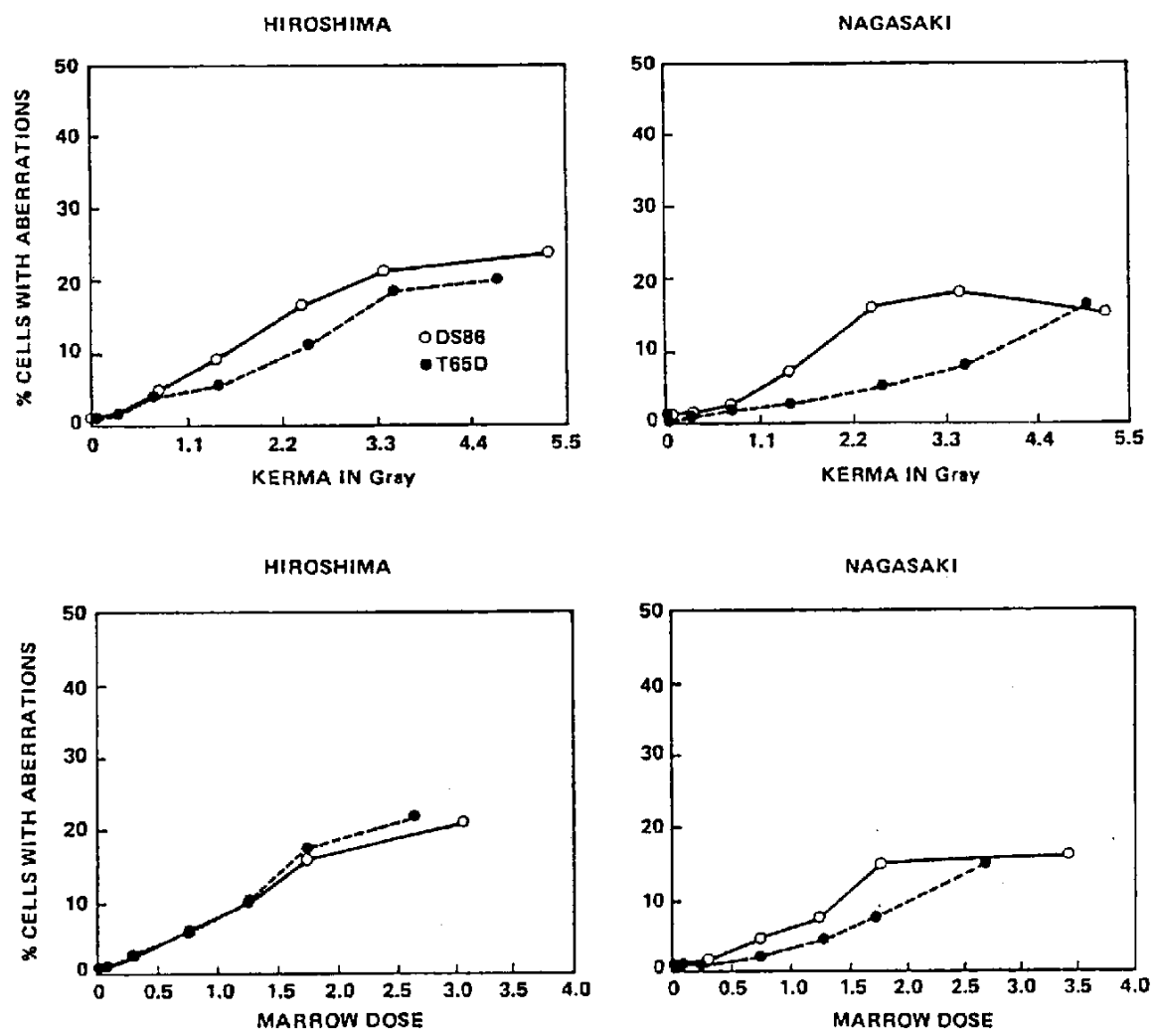

Fig. 1. Frequency of cells with chromosome aberrations in percent as a function of kerma and bone marrow dose for DS86 and T65D dosimetries, Hiroshima and Nagasaki. 
in Hiroshima. These plots also suggest that for each city, using the DS86 estimates, there are greater differences between the response functions for kerma than for total bone marrow dose (without regard to the RBE of neutron). For both DS86 and T65D, the response curves for the two cities appear to be more similar than the response curves for the two dosimetries within each city.

In the plots of DS86 data, the response appears to level off at higher estimated doses. This leveling off at DS86 kerma estimates in excess of $4 \mathrm{~Gy}$ is similar to that seen for cancer mortality data $^{7)}$ and severe epilation ${ }^{8)}$ in the Life Span Study (LSS). Although there are various reasons for flattening of the response at high doses, it is likely that errors in the dose estimates play an important role in the pattern seen here. As Jablon ${ }^{9)}$ commented regarding the impact of errors in dose estimation with respect to the T65D dosimetry, it seems possible that the true doses for survivors with high estimated dose are substantially smaller than the estimated dose. Because of concerns about the impact of such errors, samples for survivors with DS86 kerma estimates in excess of $4 \mathrm{~Gy}$ have been excluded from the present analysis. It is clear from the present data that there is appreciable overdispersion in aberration frequency between individuals within each dose group, and that the relative amount of overdispersion increases rapidly with increasing dose.

Table 2. Regreession coefficients and significance tests in linear and linear-quadratic models of the percentage of cells with chromosome aberrations

\begin{tabular}{|c|c|c|c|}
\hline \multirow{2}{*}{ Dosimetry } & \multirow{2}{*}{ Term } & \multicolumn{2}{|c|}{ Regression Coefficients } \\
\hline & & \multirow[t]{2}{*}{ Hiroshima } & Nagasaki \\
\hline & Linear moc & & \\
\hline \multirow[t]{2}{*}{ DS86 kerma } & Intercept & $1.01 \pm 0.07$ & $1.30 \pm 0.09$ \\
\hline & Linear & $5.6 \pm 0.3$ & $4.0 \pm 0.4$ \\
\hline \multirow[t]{3}{*}{ T65D kerma } & Intercept & $1.03 \pm 0.07$ & $1.28 \pm 0.09$ \\
\hline & Linear & $3.9 \pm 0.2$ & $1.9 \pm 0.2$ \\
\hline & \multicolumn{3}{|c|}{ Linear-quadratic models } \\
\hline \multirow[t]{3}{*}{ DS86 kerma } & Intercept & $1.04 \pm 0.07$ & $1.4 \pm 0.1$ \\
\hline & Linear & $3.9 \pm 0.6$ & $0.5 \pm 0.7$ \\
\hline & Quadratic & $1.0 \pm 0.3$ & $2.3 \pm 0.5$ \\
\hline \multirow[t]{3}{*}{ T65D kerma } & Intercept & $1.05 \pm 0.07$ & $1.4 \pm 0.09$ \\
\hline & Linear & $3.0 \pm 0.4$ & $-0.1 \pm 0.3$ \\
\hline & Quadratic & $0.4 \pm 0.2$ & $0.7 \pm 0.1$ \\
\hline
\end{tabular}

The intercept is an estimate of the percentage of cells with aberrations among persons whose estimated dose is 0 Gy. 
The data shown in Figure 1 suggest that linear models provide much useful information about the impact of changes in dosimetry on the percentage of cells with aberration dose-response and about inter-city differences in the response. Table 2 shows parameter estimates and the standard errors for city-specific linear response models of the form:

$$
\mathrm{Y}=\mathrm{k}+\mathrm{a} \mathrm{D}
$$

where $D$ denotes kerma in Gray and $a$, city-specific regression parameters. The parameter estimates in this table are given in terms of the percentage of cells with aberrations. Thus, for example, an intercept of 1.0 means that aberrations are seen in $1 \%$ of the cells of those NIC or zero dose survivors, while an estimated linear slope of 5.6 means that the expected percentage of aberrant cells increases by 5.6 per Gy.

The average percentage of cells with aberrations appears to increase less rapidly with dose in Nagasaki than in Hiroshima. The magnitude of the city difference in linear terms is less for the new dosimetry than with the old dosimetry. However, this difference is statistically significant for both kerma and bone marrow dose with either dosimetry.

In order to investigate nonlinearity in the dose-response function, city-specific quadratic models for the frequency of cells with chromosome aberrations were used:

$$
\mathrm{Y}=\mathrm{k}+\mathrm{a} \mathrm{D}+\mathrm{b} \mathrm{D}^{2}
$$

As with the linear models, the overdispersion parameter was assumed to be 0.55 for these models. Using the asymptotic significance level of the quadratic term in a model for both cities combined as a rough guide to the statistical significance of the nonlinearity in these models, there appear to be significant departures $(p<.001)$ from linearity with both the DS86 and T65D dosimetries for both kerma and bone marrow dose risks. Table 2 also contains parameter and standard error estimates for the city-specific models. The significance tests for the inter-city comparisons, though not shown here, parallel those for the linear model. There are significant differences in both the intercepts and the dose response. For both dosimetries, the quadratic term is steeper in Nagasaki than in Hiroshima.

\section{DISCUSSION}

\section{Other Cytogenetic Data Relevant to Dose-response Relationship}

The impact of changes in A-bomb dosimetry has caused a substantial alteration on various risk estimates for the A-bomb survivor data including the dose-response relationship for chromosome aberration frequency. As mentioned in the Results, differences in chromosome aberration frequencies still exist between Hiroshima and Nagasaki for DS86 kerma and bone marrow dose. Nevertheless, the inter-city difference in aberration frequencies observed for T65D dosimetry system becomes less pronounced for DS86 system.

In a collaborative study on the re-assessment on chromosome aberration analysis of A-bomb radiation, Sasaki ${ }^{10)}$ has reported that, although the frequencies of cells with unstable chromosome aberrations were still present with significantly high frequencies in some survivors, they were of 
limited use for the quantitative evaluation. Cells with stable aberrations showed a much clearer relationship to the ground distance from the hypocenter, indicating a positive relationship with radiation dose for individual survivors. He also pointed out that aberration frequencies in proximally exposed survivors tended to be higher in Hiroshima than in Nagasaki.

In the course of study on women who were 14-15 years of age at the time of the bombing exposed in the re-inforced concrete building (Central Telephone Office) located at a distance of 550 meters from the hypocenter in Hiroshima, Kamada et al. ${ }^{11)}$ examined cytogenetically 9 of those subjects to estimate their radiation doses. They confirmed that, in spite of very complex shielding conditions for all of these subjects, aberration frequencies were extremely high ranging from 16.7 to $41.8 \%$. This range of aberration frequency amounts to the estimated T65D kerma of at least $1.7 \mathrm{~Gy}$, being equivalent to DS86 kerma of $1.2 \mathrm{~Gy}$ or more.

\section{Data on G-band Analysis}

The majority of lymphocytes with stable chromosome aberrations are considered to have persisted since their induction in the peripheral blood of A-bomb survivors, whereas cells with dicentrics and rings have sharply decreased in frequency in the first several years after induction. Thus it seems important to use stable chromosome aberrations as a biological dosimeter particularly for those individuals who were exposed to ionizing radiation many years before their examination. Unfortunately, certain types of stable chromosome aberrations are overlooked by microscopic examination when the slides are stained with conventional Giemsa stain. Therefore, it is necessary to compare the scoring efficiency of conventional and G-banding preparations, assuming that all structural rearrangements are identifiable by the G-band analysis method.

Based on the data obtained from 23 proximally exposed Hroshima survivors, Ohtaki et al. ${ }^{12)}$ have shown that the detection rate of cells with any kind of chromosome aberrations by the conventional staining analysis is about $70 \%$ of the scoring efficiency by the G-banding method. There is, in fact, no difference in frequencies between the two methods for those whose estimated kerma was less than $1 \mathrm{~Gy}$.

G-banding analysis also provides useful information concerning accurate identification of the rearrangements on the chromosomes. This prompts the cytogeneticists to see if there are any specific breakage sites distributed nonrandomly along the entire chromosomes.

Tanaka et al. ${ }^{13)}$ have analyzed the distribution of break-points involved in radiation-induced chromosome aberrations in the lymphocytes of 39 Hiroshima A-bomb survivors, using G- and Q-band methods. The break-points in the damaged cells are found to be nonrandomly distributed both within and between the affected chromosomes; e.g., chromosomes 15,18 and 22 are involved more frequently, and 1, 2 and $\mathrm{X}$ are least involved. In contrast, using the G-banding method, Ohtaki et al. (unpublished) have observed a rather random distribution in the break-points in exchange aberrations among 23 proximally exposed Hiroshima A-bomb survivors. Thus nonrandomness in chromosome break-points may become less pronounced as more data on A-bomb survivors accumulate.

It is interesting to note that, among the stable chromosome aberrations detected by G-banding analysis on the in-utero A-bomb survivors of Hiroshima and Nagasaki, exchanges involving chromosome regions of $7 \mathrm{p} 13,7 \mathrm{q} 35,14 \mathrm{q} 11$ and $14 \mathrm{q} 32$ are observed predominantly in the distally 
exposed survivors with $0 \mathrm{~Gy}$. The break-points occurring in these specific regions are also known to be the gene loci for $\mathrm{T}$-cell receptors and immunoglobulin heavy chains.

Another notable cytogenetic feature, found in the course of screening the in-utero exposed A-bomb survivors (Ohtaki et al. unpublished), an unexpectedly high frequency of aneuploid cells due to loss or gain of an X chromosome in females, and similarly a high frequency of preferential loss and gain of chromosome 21 in either sex. In general, as chromosome size decreases, the aneuploid cell frequency increases. Provided the expected values for aneuploidy as inversely proportional to relative chromosome length, there is in good agreement between observed and expected values for all but chromosomes 21 and $\mathrm{X}$. These results indicate that both loss and gain of the $\mathrm{X}$ chromosome in females and chromosome 21 in both sexes may have much less selective disadvantage to cells for survivability and proliferative capacity in vivo.

\section{Overdispersion in aberration frequencies}

It is known that there is a striking variability of the frequency of cells with chromosome aberrations between survivors within a given dose category both for DS86 and T65D dosimetries as well as for both cities. Since a detailed account on the problem of overdispersion in chromosome aberration frequencies will be given elsewhere in this volume, the results of statistical analysis by Sposto et al. ${ }^{14)}$ will be summarized briefly as follows.

The dose-response relationship for aberration frequency using the DS86 assigned dose is signifiçantly steeper in the subsample of survivors manifesting severe epilation after the bomb than those without severe epilation. in addition, there is substantially more variation between individuals at higher doses in the proportion of cells with stable chromosome aberrations than would be expected if there were no heterogeneity in the dose-response between individuals. These findings lead to the suggestion that random dosimetry errors in the range from 45 to $50 \%$ of true dose can explain the difference in dose-response between severe and non-severe epilation groups. This amount of dosimetry error also accounts for the overdispersion in chromosome aberration frequencies.

In relation to an overdispersion in aberration frequencies in A-bomb survivors, it is noteworthy to mention here the presence of "cytogenetic outliers" whose aberration frequencies are grossly discrepant with regard to estimated dose; i.e., individuals with high aberration frequencies in the low dose range, and with low frequencies at high doses. While they may indicate differential radiation sensitivity in A-bomb survivors, and other interpretations are readily possible, some biological endpoints studied recently at RERF indicate that no difference in radiosensitivity is demonstrable among A-bomb survivors. (See other papers in this issue.)

A mean frequency of dicentrics (data not shown here) in the O-dose group, including those who are in the NIC group, seems appreciably high when compared with reported evidence (Bender et al. ${ }^{15)}$ ). They differ by a factor of $2 ; 2.5 \times 10^{-3}$ in our data as opposed to $1.3 \times 10^{-3}$ in other study populations. One of the possible interpretations for this difference is that the majority of survivors have received substantial amounts of medical X-ray radiation for diagnostic purposes, especially fluoroscopy of the upper gastro-intestinal region, more frequently than non-exposed people of the same age, because of the better medical care extended to A-bomb survivors.

It seems essential to establish an in vivo dose-response relationship for chromosome 
aberrations of both stable and unstable types for survivors repeatedly exposed to low dose diagnostic X-ray exposure for prolonged period of time.

\section{Possible Future Direction in Cytogenetic Studies}

Since there still exists a difference in dose-response relationship for chromosome aberrations between Hiroshima and Nagasaki, an estimation of the relative biological effectiveness (RBE) of neutron radiations will be one of the most important tasks to be investigated in the near future using every possible biological endpoint based on A-bomb survivor data. Thus it becomes important to obtain more accurate data on persistent chromosome aberrations of the stable type, mostly reciprocal translocations, in A-bomb survivors. To do this, the introduction of molecular biological techniques for the detection of structural rearrangements of chromosomes in the affected lymphocytes of survivors is under way.

One of the technologies readily available to date is known as "chromosome painting" technique recently developed by Lucas et al. ${ }^{16}$. This is a new technique to differentially stain specific chromosomes using fluorescence in situ hybridization with chromosome specific composite probes. In order to establish a better biological dosimetry system with chromosome aberration data, the present "chromosome painting" technique is obviously advantageous for the accurate and extensive screening of specific reciprocal translocations that are still observable as the major contributor to the dose-response relationship.

\section{ACKNOWLEDGMENTS}

I am very much indebted to Dr. Seymour Abrahamson, Chief of Research, Permanent Director of RERF, for his advice and keen interest to this study, and for going through the manuscript. I am grateful to Dr. M. Nakano, Mr. K. Ohtaki, Mr. Y. Kodama, and all of the staff members in Hiroshima Cytogenetics Laboratory, and to Dr. T. Honda, Mr. M. Itoh and all technical staff members in Cytogenetics Laboratory, Department of Radiobiology, Nagasaki, for their continuous cooperation and technical assistance, and also to Mrs. M. Utaka for her clerical help.

\section{REFERENCES}

1. Awa, A.A. (1975) Review of thirty years study of Hiroshima and Nagasaki atomic bomb survivors. II. Biological effects. G. Chromosome aberrations in somatic cells in somatic cells. J. Radiat. Res. 16(Suppl): 122-131.

2. US-Japan joint reassessment of atomic bomb radiation dosimetry in Hiroshima and Nagasaki. Final Report (1987) Vol. 1 and 2 (ed. Roesch, W.C.) Radiation Effects Research Foundation.

3. Awa, A.A., Sofuni, T., Honda, T., Itoh, M., Neriishi, S. and Otake, M. (1978) Relationship between the radiation dose and chromosome aberrations in atomic bomb survivors of Hiroshima and Nagasaki. J. Radiat. Res. 19: 126-140.

4. Awa, A.A. (1988) Chromosomal aberrations in atomic bomb survivors. In Genetics of human tumors in 
Japan. Gann monograph on cancer research No. 35 (Takebe, H., Utsunomiya, J., eds) pp.175-189, Jap. Sci. Soc., Tokyo.

5. Awa, A.A., Ohtaki, K., Itoh, M., Honda, T., Preston, D.L. and McConney, M.E. (1988) Chromosome aberration data for A-bomb dosimetry reassessment: Proceedings of the 23rd annual meeting of NCRP, 8-9 April 1987, Washington DC, Proc No. 9, pp.185-202.

6. Preston, D.L., McConney, M.E., Awa, A.A., Ohtaki, K., Itoh, M. and Honda, T. (1988) Comparison of the dose-response relationships for chromosome aberration frequencies between the T65D and DS86 dosimetry. RERF Tech. Rep. 7-88.

7. Preston, D.L. and Pierce, D.A. (1987) The effect of changes in dosimetry on cancer mortality risk estimate in the atomic bomb survivors. RERF Tech. Rep. 9-87.

8. Stram, D.O. and Mizuno, S. (1988) Analysis of the DS86 atomic bomb radiation dosimetry methods using data on severe epilation. RERF Tech. Rep. 1-88.

9. Jablon, S. (1988) Atomic bomb radiation dose estimation at ABCC. ABCC Tech. Rep. 23-71.

10. Sasaki, M.S. (1988) Chromosomal approaches to the dose assessment in human exposures to ionizing radiation. Berzelius Symp. XV, pp.119-128.

11. Kamada, N., Shigeta, C., Kuramoto, A., Munaka, M., Yokoro, K., Niimi, M., Aisaka, C., Ito, C. and Kato, H. (1989) Acute and late effects of A-bomb radiation studied in a group of young girls with a defined condition at the time of bombing. J. Radiat. Res. 30: 218-225.

12. Ohtaki, K., Shimba, H., Awa, A.A. and Sofuni, T. (1982) Comparison of type and frequency of chromosome aberrations by conventional and G-staining methods in Hiroshima atomic bomb survivors. J. Radiat. Res. 23: 441-449.

13. Tanaka, K., Kamada, N., Ohkita, T. and Kuramoto, A. (1983) Nonrandom distribution of chromosome breaks in lymphocytes of atomic bomb survivors. J. Radiat. Res. 24: 291-304.

14. Sposto, R., Stram, D.O. and Awa, A.A. (1990) An investigation of random errors in the DS86 dosimetry using data on chromosome aberrations and severe epilation. RERF Tech. Rep. 7-90.

15. Bender, M.A., Awa, A.A., Brooks, A.L., Evans, H.J. Groer, P.G., Littlefield, L.G., Pereira, C., Preston, R.J., Wachholz, B.W. (1988) Current status of cytogenetic procedures to detect and quantify previous exposures to radiation. Mutat. Res. 196: 103-159.

16. Lucas, L.N., Tenjin, T., Straume, T., Pinkel, D., Moore, D. II, Litt, M. and Gray, J.W. (1989) Rapid human chromosome aberration analysis using fluorescence in situ hybridization. Int. J. Radiat. Biol. 56: 35-44. 